\title{
A Comparative Analysis of Mathematical and Linear Regression Models to Predict the Outcomes of COVID-19 Pandemic in Rwanda
}

\author{
Gratien Twagirumukiza ${ }^{1, ~ *}$, Edouard Singirankabo ${ }^{2}$, Leopord Hakizimana ${ }^{3}$ \\ ${ }^{1}$ Faculty of Applied Fundamental Sciences, Institute of Applied Sciences, Musanze, Rwanda \\ ${ }^{2}$ School of Mathematics, Jomo Kenyatta University of Agriculture and Technology, Nairobi, Kenya \\ ${ }^{3}$ School of Computing and Information Technology, University of Kigali, Musanze, Rwanda
}

Email address:

gratien@aims.edu.gh (G. Twagirumukiza), singirankabo.edouard@student.jkuat.ac.ke (E. Singirankabo),

lhakizimana@uok.ac.rw (L. Hakizimana)

${ }^{*}$ Corresponding author

\section{To cite this article:}

Gratien Twagirumukiza, Edouard Singirankabo, Leopord Hakizimana. A Comparative Analysis of Mathematical and Linear Regression Models to Predict the Outcomes of COVID-19 Pandemic in Rwanda. Mathematics and Computer Science. Vol. 6, No. 5, 2021 , pp. 77-82. doi: $10.11648 /$ j.mcs.20210605.12

Received: August 30, 2021; Accepted: October 12, 2021; Published: October 28, 2021

\begin{abstract}
The research objective was to investigate the level of COVID-19 outbreak in Rwanda using mathematical and linear models for future prediction of the disease. Both Mathematical model and linear model were used. A sequential mathematical preliminary of COVID-19 was considered to check how it grows within a large number of population. The model diagram was proposed with four compartmental model. The non-linear dynamical system of COVID-19 was derived from the model. The model was checked for positivity and boundedness in system. We found that it's positively invariant in system. The results also showed that the disease is locally and globally unstable due to the fact that the basic reproduction number is greater than zero i.e., $R_{0}>0$. The basic reproduction number was computed using the next generation Matrix and found that COVID-19 affects a very large population in the system. Method for real data: The study used a sample of 463 COVID-19 daily reports, that is, the available data by 9 April 2021. The data are analyzed using Statistical software (STATA version 13.1). The probability of skewness and kurtosis was $P \leq 0.0001$ for New cases, and New deaths. Besides Chi-Square $p$ $\leq 0.0001$ for both New cases and New deaths was $<0.05$ that means the significance at a $5 \%$ level. Results: By comparing the mean and standard deviation, the results show that the number of New cases is higher than that of New deaths, that is 50.00432 with high standard deviation 78.47841 , and 0.6781857 with low standard deviation 1.474935 ; respectively. A spearman rank correlation shows strong correlation between New cases and New deaths. Linear regression analysis model shows that there is a linear relationship of New cases with New deaths. The findings show that the number of deaths will be higher than New cases. Conclusion: The statistics show that COVID-19 is still there within individuals and is moving around. The findings show that in future, the number of new deaths will be higher than that of new cases at a time $t$. We recommend the government of Rwanda to speed up the Vaccination to the total population to avoid more future deaths due to COVID-19 and to strictly tightening the preventive measures for both Rwandans and incoming travelers. With the above mentioned strategies and the measures, there is a hope that If the whole country is vaccinated, COVID-19 will vanish at time $t$.
\end{abstract}

Keywords: COVID-19, Comparative Analysis, Mathematical Model, Regression Model, Outbreak Prediction

\section{Introduction}

COVID-19 is an infectious disease caused by severe acute respiratory syndrome known as Corona virus. It is transmitted from one person to another [1]. According to the current situation in Rwanda and worldwide, COVID-19 is primarily spread between people through respiratory droplets and contact routes [2] and; Some of its common symptoms include but not limited to fever, shortness of breath and dry cough. COVID-19 has been a challenge up to now where 
many countries mildly started many different preventive measures to curb its spread. Nowadays, COVID-19 is the leading cause of deaths worldwide where it kills more than 5 thousand people/day with over 400 thousand new cases/day on the globe [3]. It's been over 1 year since COVID-19 occurred. It was first reported in China in December 2019 in the city of Wuhan, the capital of Hubei Province [4], and by 30 March 2021, World Health Organization (WHO) report shows that the cumulative deaths in percentages were $48 \%$ in Americas, 34\% in Europe, 8\% in South-East Asia, 6\% in Eastern Mediterranean, 3\% in Africa, and 1\% in Western Pacific; with new cases 44\%, 34\%, 12\%, 6\%, 3\%, and $1 \%$ respectively. The $\mathrm{WHO}$ report shows that over 2.5 millions of people died with COVID-19 globally with over 115 millions of newly infected cases [3]. According to WHO report in Africa; Africa's first COVID-19 case was recorded in Egypt on 14 February 2020 from an adult male whose 17 contacts tested negative and prompted African preparedness efforts. COVID-19 cases in Africa were mostly imported from Europe due to the higher volume of business and tourism airline traffic between African countries, Europe, and less from China [5, 6]. The first COVID-19 case in Rwanda was first recorded on 14 March 2020 and reported by the Ministry Of Health $(\mathrm{MOH})$ where Rwandan government has started the preventive measures against COVID-19 [7]. The registered cases have been dramatically increasing and the government began to tight the measures. In this context, schools have stopped and followed by the total lock-down for almost 7 months. A very few number of research on the impact of COVID-19 in Rwanda were done but none has ever tried the comparative models to predict the future behavior of COVID-19 in Rwanda. Mathematical models have been used over a decade to predict the disease outbreak in epidemiology. However, linear models can be used in statistical data analysis to predict future behavior of a disease based on the past and current situation. In this research, we used a comparative analysis of the two models to predict the impact of COVID-19 global pandemic in Rwanda.

A sequential mathematical preliminary of COVID-19 growth in Rwanda.

If one person is confirmed COVID-19 positive with lab test in Rwanda, s/he may contact the minimum of 3 persons within that day and the symptoms can be seen within 14 days. If this is the case, to find how many persons that $\mathrm{s} / \mathrm{he}$ can spread COVID-19 in 14 days; consider

$$
a=1, r=3, n=14 \text {, }
$$

The total number of people to be affected is calculated as follows:

$$
\begin{aligned}
S_{n}= & a\left(r^{n}-1\right) /(r-1) \\
S_{14}= & 1\left(3^{14}-1\right) /(3-1) \\
= & 4,782,968 / 2 \\
& =2,391,484
\end{aligned}
$$

If number of cases registered is 5, then in 14 days,

$$
\begin{aligned}
S_{n}= & 5\left(r^{n}-1\right) /(r-1) \\
S_{14}= & 5\left(3^{14}-1\right) /(3-1) \\
= & 23,914,840 / 2 \\
& =11,957,420
\end{aligned}
$$

\section{Mathematical Model}

This model is composed by four compartments namely susceptible class $(S(t))$, Infected class $(I(t))$, Recovered class $(R(t))$, and Death's class $(D(t))$. Having noticed that there is no clear treatment of COVID-19, we assume that some people can be recovered time to time due to their body immunity and in this context, we assume that there is no vaccine trusted $100 \%$ to help people so that the vaccinated ones cannot get back an infection at time $t$. Referring to SITR model with treatment introduced by [8], the following non linear dynamical system was derived to form SIRD model at time $t$.

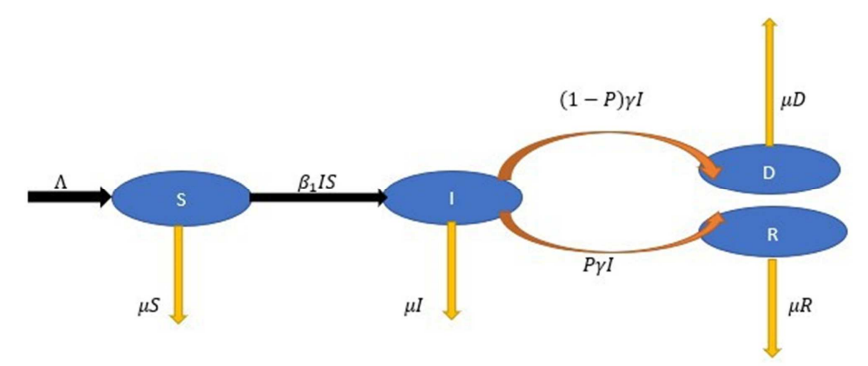

Figure 1. A proposed schematic diagram of COVID-19 mobility in Rwanda.

The proposed schematic Figure 1 of COVID-19 in Rwanda, shows the mobility of the disease. The recruitment rate $\Lambda$ is considered as the mobility of people outside entering the system. The susceptible population class is considered to be normal population and we assume that everyone can be infected at a time $t$. In this context, there is a contact between susceptible and infected ones with $\beta_{1}$ contact rate. For the infected individuals there is a probability $P$ that some infected individuals can get recovered due to there immunity and/or $(1-P)$ otherwise die. We assume that there is no treatments and/or vaccines trusted in this case. $\gamma$ is the rate at which individuals get recovered due to the body immunity and $\mu$ stands for natural death not derived from COVID-19; in all compartments. The following is non linear dynamical system of equations derived from the diagram;

$$
\begin{gathered}
d S / d t=\Lambda-\left(\beta_{1} I+\mu\right) S \\
d I / d t=\beta_{1} I S-(\mu+\gamma) I \\
d R / d t=P \gamma I-\mu R \\
d D / d t=(1-P) \gamma I-\mu D
\end{gathered}
$$

Subjected to the non negative initial conditions $S=S_{0} \geq 0, I=$ $I_{0} \geq 0, R=R_{0} \geq 0, D=D_{0} \geq 0$. 


\subsection{Positivity of the System}

The associated parameters of the system (2) with respect to the initial conditions are non negative for all $t>0$.

Lemma

If $\{S(0), I(0), R(0), D(0)\}$ and all associated parameters of the system are positive, then solutions $S(t), I(t), R(t), D(t)$ are all positive $\forall t>0$.

Proof 2.1 Let's consider the following assumption for ordinary non linear differential equations of the model 2 ,

$$
t_{1}=\sup \{t>0: S(t)>0, I(t) \geq 0, R(t) \geq 0, D(t) \geq 0\}
$$

From the first equation of the model (2), the following equation holds

$$
\begin{array}{cc}
d S / d t=\Lambda-\beta_{1} I S-\mu S & 0, I=I_{0} \geq 0, R=R_{0} \geq 0, D=D_{0} \geq 0 ; \text { we get: } \\
I\left(t_{1}\right) \geq I(0)+e^{-(\mu+\gamma) t 1}>0 ; R\left(t_{1}\right) \geq R(0)+e^{-\mu t 1}>0 ; D\left(t_{1}\right) \geq D(0)+e^{-\mu t 1}>0 \text { respectively. }
\end{array}
$$

Since $\Lambda \geq 0$, we can write the rest in the following form; then

$$
d S / d t \geq-(\mu+\theta) S,
$$

with $\theta=B_{1} I$

$$
\begin{gathered}
\int_{0}^{t_{1}} \frac{d S}{S} \geq-\int_{0}^{t_{1}}(\mu+\theta) d t \\
\ln |S(t)|_{0}^{t_{1}} \geq-(\mu+\theta) t_{1}, \\
|S(t)|_{0}^{t_{1}} \geq e^{-(\mu+\theta) t_{1}}, \\
S\left(t_{1}\right) \geq S(0)+e^{-(\mu+\theta) t_{1}}>0 .
\end{gathered}
$$

By using the same approach with (4), with the fact $S=S_{0} \geq$

\subsection{Boundedness of the System}

The rate at which the total population varies over time is given by:

$$
\begin{gathered}
d N / d t=d S / d t+d I / d t+d R / d t+d D / d t \\
=\Lambda-\left(\beta_{1} \mathrm{I}+\mu\right) \mathrm{S}+\beta_{1} \mathrm{IS}-(\mu+\gamma) \mathrm{I}+\mathrm{P} \gamma \mathrm{I}-\mu \mathrm{R}+(1-\mathrm{P}) \gamma \mathrm{I}-\mu \mathrm{D}=\Lambda-\mu(\mathrm{S}+\mathrm{I}+\mathrm{R}+\mathrm{D})
\end{gathered}
$$

Since the total population $N=S+I+R+D$, then the following equation (5) holds to check the boundedness

$$
d N / d t=\Lambda-\mu N
$$

Lemma:

The closed set

$$
\zeta=\{S, I, R, D\} \in \mathbb{R}_{+}^{4}: 0 \leq(S+I+R+D) \leq \frac{\Lambda}{\mu}
$$

positively invariant.

Proof 2.2 Assume that

$\{S(t), I(t), R(t), D(t)\} \in \mathbb{R}_{+}^{4}, \forall t>0$. The equation (5) can be written as

$$
\begin{aligned}
& \frac{d N}{\Lambda-\mu N}=d t \\
& \int_{0}^{t} \frac{d N}{\Lambda-\mu N}=\int_{0}^{t} d t \\
& N(t)=N(0) e^{-\mu t}+\frac{\Lambda}{\mu}\left(1-e^{-\mu t}\right) ; \lim _{t \rightarrow \infty} N(t)=\frac{\Lambda}{\mu^{\prime}} \\
& \mathcal{F}=\left[\begin{array}{c}
\beta_{1} I S \\
0
\end{array}\right] ; \operatorname{Jacobian}(\mathcal{F})_{\text {Disease free }}(F)=\left[\begin{array}{cc}
\frac{\Lambda \beta_{1}}{\mu} & 0 \\
0 & 0
\end{array}\right] \\
& v=\left[\begin{array}{c}
(\mu+\gamma) I \\
-P \gamma I+\mu R
\end{array}\right] ; \operatorname{Jacobian}(v)_{\text {Disease free }}(V)=\left[\begin{array}{cc}
\mu+\gamma & 0 \\
-P \gamma & \mu
\end{array}\right] \\
& F V^{-1}=\left[\begin{array}{cc}
\frac{\Lambda \beta_{1}}{\mu} & 0 \\
0 & 0
\end{array}\right]\left[\begin{array}{cc}
\frac{1}{\mu+\gamma} & 0 \\
\frac{P \gamma}{\mu(\mu+\gamma)} & \frac{1}{\mu}
\end{array}\right]=\left[\begin{array}{cc}
\frac{\Lambda \beta_{1}}{\mu(\mu+\gamma)} & 0 \\
0 & 0
\end{array}\right]
\end{aligned}
$$
the next generation matrix [10] and it is then given by $R_{0}=\rho\left(F V^{-1}\right)$ with $\rho(A)$ denotes the spectral radius of a matrix $A, F$ the new infection transfer terms, $V$ is the non singular matrix of the remaining

If $N(0) \leq \frac{\Lambda}{\mu}$ then we have $N(t) \leq \frac{\Lambda}{\mu}, \forall t>0$. Furth
If $N(0)>\frac{\Lambda}{\mu}$, then the solutions $(\mathrm{S}(\mathrm{t}), \mathrm{I}(\mathrm{t}), \mathrm{R}(\mathrm{t}), \mathrm{D}(\mathrm{t}))$.

enter the closed set $\zeta$ which affirms that $\zeta$ is positively invariant. So, the region $\zeta$ contains all solutions in $\mathbb{R}_{+}^{4}$.

Hence, It is sufficient to study the disease transmission Basic reproduction number $R O$

This is defined as the number of secondary infected individuals caused by only one infected person and it is a threshold parameter that shows the disease behaviors to see whether the disease stays or wipes out of population 


\section{Parameters estimation and Numerical simulation}

This subsection gives the parameter estimates of COVID-19 as per 9 April 2021 in Rwanda; of the proposed model (2). We used Python 3 programming language to carry out this Analysis with the following initial conditions:

$$
S=13168393, I=23260[11], R=20991[11], D=314
$$

[11] which comes to the total of $N=13,212,958$ The current population of Rwanda as of Tuesday, April 27, 2021 [12], based on Worldometer elaboration of the latest United Nations data. By subtracting the total population with the Recovered $(R)$, Infected $(I)$ and Deaths $(D)$; we obtained the right susceptible population. The parameter values are in table 1 below.

Table 1. Parameter values and their description used.

\begin{tabular}{llll}
\hline Parameters & Description & Value/days & Source \\
\hline$\Lambda$ & Recruitment rate & 200 & Estimated \\
$\beta_{1}$ & Effective contact rate & 0.00093 & {$[8]$} \\
$P$ & Proportion of infected individuals joining either the class $R$ or $D$ & 0.04 & {$[8]$} \\
$\gamma$ & Rate of Recovered individuals due to their immunity & 0.4 & {$[13]$} \\
$\mu$ & The natural death rate in all compartments & 0.063 & {$[8]$} \\
\hline
\end{tabular}

Simulation Results of predicted new cases and Deaths based on the current situation.
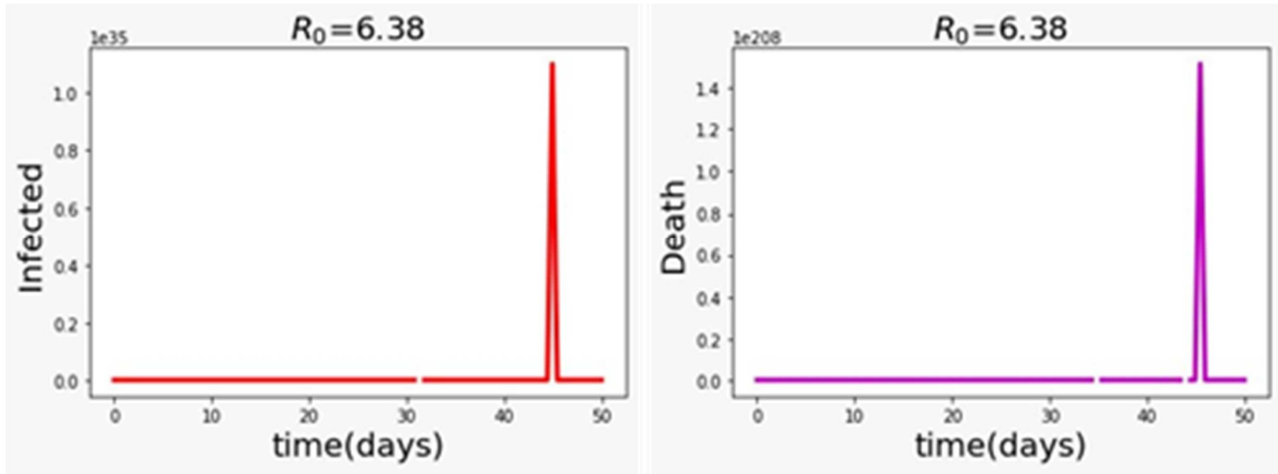

Figure 2. Prediction of Infected Individuals (New cases) Vs Deaths (New Deaths) at time $t=45$ days.

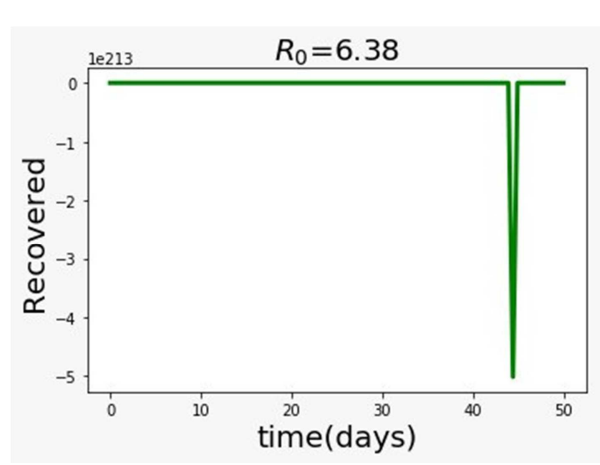

Figure 3. Prediction of Recovered individuals at time $t=45$ days.

\section{Linear Model}

In this section, we have used STATA 13.1 software to investigate COVID-19 outbreak in Rwanda for future prediction. We also found the association and linear relationship between the New COVID-19 cases and New COVID-19 deaths using the spearman rank correlation coefficient and the linear regression model of the extracted data available at Rwanda Biomedical Centre (RBC) [14] as per 9 April 2021 and from the available data, the first incidence of COVID-19 in Rwanda was recorded on 14 February 2020 introduced by one individual and started to spread.

\begin{tabular}{r|rrr} 
Source & SS & df & US \\
\hline $\begin{array}{r}\text { Hodel } \\
\text { Residual }\end{array}$ & 3013572.21 & 2 & 1506786.11 \\
5257459.79 & 460 & 11429.2604 \\
\hline Total & 0271032 & 462 & 17902.6667
\end{tabular}

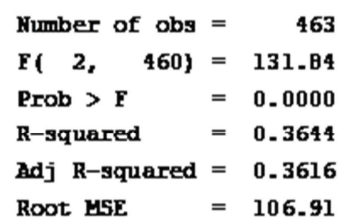

Root HSE $\quad=106.91$

\begin{tabular}{r|rrrrrr}
\hline Date_repor-d & Coef. & Std. Err. & $t$ & P $>|t|$ & [958 Conf. Interval] \\
\hline Hew_cases & .8455826 & -0914859 & 9.24 & 0.000 & -6658005 & 1.025365 \\
Hew_deaths & 12.57506 & 4.867786 & 2.58 & 0.010 & 3.00921 & 22.14092 \\
_cons & 22097.19 & 5.89312 & 3749.66 & 0.000 & 22085.61 & 22108.77 \\
\hline
\end{tabular}

Figure 4. COVID-19 in Rwanda characteristics mean and Standard deviation. 


\begin{tabular}{r|rrrrr} 
Variable & Obs & Hean & std - Dev - & Hin & Max \\
\hline Ned_cases & 463 & 50.00432 & 78.47841 & 0 & 574 \\
Hey_deaths & 463 & -6781857 & 1.474935 & 0 & 11
\end{tabular}

Figure 5. Linear regression model for COVID-19 in Rwanda characteristics.
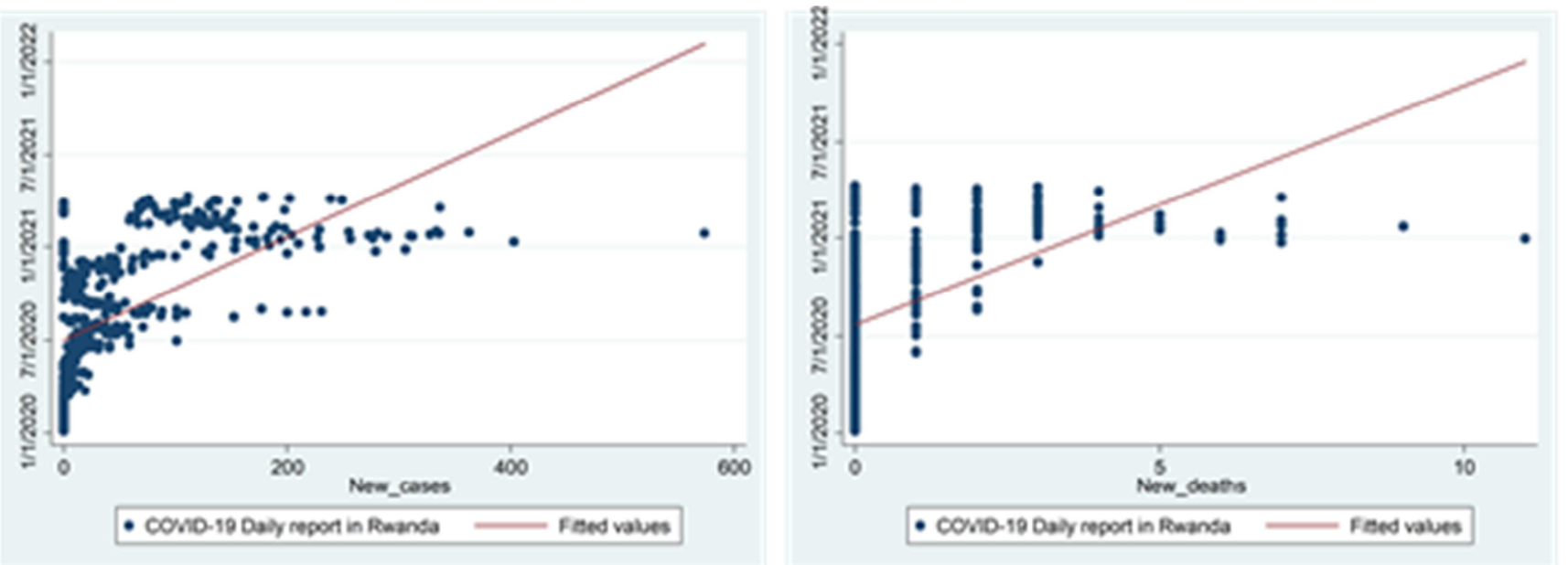

Figure 6. New cases and New deaths scatter plot against COVID-19 daily report as for 9 April 2021.

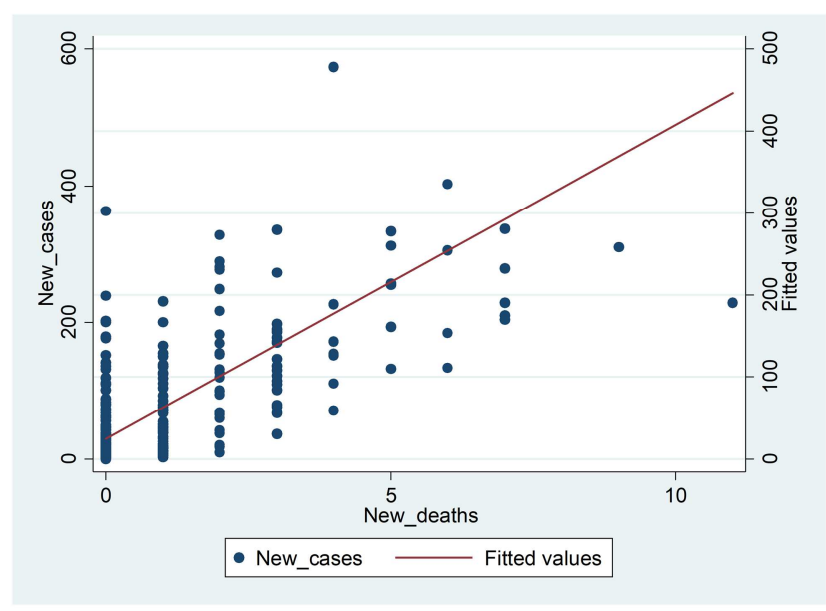

Figure 7. New deaths against New cases.

\section{Results and Discussions}

The study was about investigating the level of COVID-19 outbreak in Rwanda using mathematical and linear models for future prediction of the disease. The study used both models. A sequential mathematical preliminary of COVID-19 in Rwanda was conducted. We have seen that if 1 individual is tested positive, can atleast meet 3 people within a day. In this context, the basic reproduction number would be greater than or equal to three $R_{0} \geq 3$ which comes to the domain $D=$ $[3,+\infty]$. From this estimated $R_{0}$, we have computed $R_{0}$ using the next generation matrix to see whether the disease can spread in a domain. The result shows that the basic reproduction number in equation (7) equals to 6.3766 which is in the estimated domain. The Figure 2 shows the prediction of Infected individuals with new deaths at time $t=45$ days based on the current situation. The plots show that the disease will remain steady in system up to 44 days for both newly infected and new deaths. Furthermore, the plots show that the number of deaths will be greater than that of new cases within each 45 days at the current basic Reproduction number of $R_{0}=6.38$ with the assumed recruitment rate of atleast the estimated 200 recruited individuals based on the current data as per 9 April 2021. From Figure 3, the plot shows that within 45 days at the same parameters, the recovered individuals is predicted to dramatically decrease. The Figures 3 and 2 indicate that we can expect a high decrease in recovered people and increase in deaths at time $t=45$ days for the same parameters. Based on this $R_{0}$, this is an implication that a very large number of population in Rwanda has been affected but due to very strong immunity for some people the disease vanishes; however those with very weak immunity die. In figure 4 , the statistics show that mean and standard deviation for New cases are higher than that of New deaths. These results show that the number of infected individuals is greater than the number of deaths. The figure 5 shows linear regression model for both New cases and New deaths. By determining how well the model fits, the coefficient of determination value was 0.3644 which explains $36.44 \%$ of the variability of COVID-19 daily reports. F-ratio test was computed and output shows that the independent variables statistically significantly predict the dependent variable and the regression model is a good fit of the data. For the estimated model coefficients, the general form of the equation to predict COVID-19 from New cases, and new deaths is:

Predicted COVID -19 in Rwanda $=22097.19+(.8455826 *$ Newcases $)+(12.57506 *$ Newdeaths $)$ 
Which is the regression model needed. The model shows that for each 1 increase in Newcases, there is an increase in COVID-19 in Rwanda of .8455826 and for each 1 increase in Newdeaths, there is an increase in COVID-19 in Rwanda of 12.57506. The findings show that in future, the number of new deaths will be higher than that of new cases at a time $t$. The coefficients are statistically significant as per corresponding $P-$ Values $<0.05$ the standard significance level at $95 \%$ confidence interval.

\section{Conclusion}

The statistics show that COVID-19 is still there within individuals and is moving around. The findings show that in future, the number of new deaths will be higher than that of new cases at a time t. We recommend the government of Rwanda to speed up the Vaccination to the total population to avoid more future deaths due to COVID-19 and to strictly tightening the preventive measures for both Rwandans and incoming travelers. With the above, there is a hope that If the whole country is vaccinated, COVID-19 will vanish at time $t$.

\section{Real Data Availability}

The real data used in this study can be seen on Rwanda Biomedical Centre website (www.rbc.gov.rw) Situation Report on COVID-19 from the first registered case, that is, 14 March 2020 to 9 April 2021 reporting period.

\section{Conflict of Interest}

The authors declare that they have no competing interests.

\section{References}

[1] Joab O Odhiambo, Philip Ngare, Patrick Weke, and Romanus Odhiambo Otieno. Modelling of COVID-19 transmission in kenya using compound poisson regression model. Journal of Advances in Mathematics and Computer Science, pages 101111,2020

[2] World Health Organization et al. Modes of transmission of virus causing COVID-19: implications for ipc precaution recommendations: scientific brief, 27 march 2020. Technical report, World Health Organization, 2020.
[3] World Health Organization et al. COVID-19 weekly epidemiological update, 16 march 2021. 2021.

[4] Gianfranco Spiteri, James Fielding, Michaela Diercke, Christine Campese, Vincent Enouf, Alexandre Gaymard, Antonino Bella, Paola Sognamiglio, Maria Jose Sierra Moros, Antonio Nicolau Riutort, et al. First' cases of coronavirus disease 2019 (COVID19 ) in the who european region, 24 january to 21 february 2020. Eurosurveillance, 25 (9): 2000178, 2020.

[5] Shaheen Mehtar, Wolfgang Preiser, Ndeye Aissatou Lakhe, Abdoulaye Bousso, Jean-Jacques Muyembe` TamFum, Oscar Kallay, Moussa Seydi, Alimuddin Zumla, and Jean B Nachega. Limiting the spread of COVID-19 in africa: one size mitigation strategies do not fit all countries. The Lancet Global Health, 8 (7): e881-e883, 2020.

[6] World Health Organization et al. Coronavirus disease 2019 (COVID-19): situation report, 82. 2020.

[7] Alfred Bizoza and Simeon Sibomana. Indicative socioeconomic impacts of the novel coronavirus (COVID-19) outbreak in eastern africa: Case of rwanda. Available at SSRN $3586622,2020$.

[8] Ebenezer Bonyah, Gratien Twagirumukiza, and Patience Pokuaa Gambrah. Mathematical analysis of diarrhoea model with saturated incidence rate. 2019.

[9] Odo Diekmann, Johan Andre Peter Heesterbeek, and Johan AJ Metz. On the definition and the computation of the basic reproduction ratio $\mathrm{r} 0$ in models for infectious diseases in heterogeneous populations. Journal of mathematical biology, 28 (4): 365-382, 1990.

[10] Pauline Van den Driessche and James Watmough. Reproduction numbers and sub-threshold endemic equilibria for compartmental models of disease transmission. Mathematical biosciences, 180 (1-2): 29-48, 2002.

[11] Cumulative number in Rwanda. Update COVID-19 09 april 2021. WHO, https://www.afro.who.int/news/update-COVID19-09-april-2021, Accessed April 2021.

[12] Total population, 2021. Rwanda population (live). worldometer, https://www.worldometers.info/worldpopulation/rwanda-population/, Ac-cessed April 2021.

[13] Gratien Twagirumukiza and Edouard Singirankabo. Mathematical analysis of a delayed hiv/aids model with treatment and vertical transmission. Open J. Math. Sci, 5: 128-146, 2021.

[14] Situation Report on COVID-19. Situation report on novel coronavirus. Rwanda Biomedical Centre, https://www.rbc.gov.rw/index.php?id=717, Accessed April 2021. 